\title{
The missing links for a productive work/life balance
}

\section{Opinion}

Sleep deprived? Under nourished? Sedentary lifestyle? These are all factors that can directly affect our productivity in the workplace, at home, and in our personal and professional life.

"According to the Mayo Clinic, a healthy diet can not only help you control your weight and lower your cholesterol, but it can also improve your concentration, alertness, problem-solving skills, and productivity."

"According to the Centers for Disease Control and Prevention, approximately one-third of U.S. adults are obese. These growing obesity rates not only lead to increases in diabetes and heart disease, but also to increased costs in the workplace due to decreased productivity."

"The UCLA Health System reports that poor diet habits can lead to increased fatigue, decreased mental effectiveness, increased irritability, decreased energy levels, decreased ability to think clearly, decreased ability to perform your job effectively, high levels of stress and depression, and decreased productivity."

Food is a powerful thing! Adding nutrient-dense super foods to your daily diet may help to boost our immune system improve energy and enhance our performance making us more productive in our everyday lives. Superfoods like wheatgrass, cod liver oil, fruits, vegetables, nuts, seeds and ancient grains naturally provide our bodies and brains with fuel. These foods are alkalizing on the body helping to reduce inflammation and improve mineral balance. When we don't sleep, eat processed foods, and have high stress, our body becomes very acidic leaching out valuable minerals including calcium, iodine and magnesium. Overtime, this can cause problems with our sleep, hormones, and overall health. Reducing inflammation by adding super foods can help clear our minds and enhance concentration making room for positive and productive thoughts. Of course it's not enough to just eat healthy. Incorporating rest and movement practices into the equation are essential for health and productivity.

We all know how sleep can affect us. A poor night often results in complaints of low energy, poor work performance, and irritability. Lack of sleep is the fastest way to age the human body. Not only does it break down our bodies but it also weakens our minds. Lack of sleep causes us to age at a much faster rate contributing to weaker bones, wrinkles, and hormonal imbalances. Getting seven to eight hours of sleep each night is essential for improved health and increased productivity. This is because it takes over 5hours to hit the crucial stages of our sleep cycle that causes our bodies to release healing hormones contributing to better energy and a healthier weight. REM sleep or our "dream state," occurs during the final stage of our sleep cycle. This is when the brain is in healing mode and where long-term memories are stored.

Did you know that some ongoing studies are actually considering whether adequate sleep should be a standard part of weight loss programs? "According to the Healthy Living Huff Post,
Volume 2 Issue 3 - 2015

\author{
Kristen Johnson \\ Mrs. Green's Natural Market, USA
}

Correspondence: Kristen Johnson, Mrs. Green's Natural Market, I4I5 North Dearborn Unit I5D, Chicago, IL 606I0, USA, Tel 5172304528, Email kristenj33@gmail.com

Received: June 18,2015 | Published: July 25, 2015

Epidemiological studies have shown that disturbed sleep leads to increased weight gain."

To avoid disturbed sleep, it's important to limit stimulating blue light from tech devices. Technology is a natural energy drainer and can suppress melatonin, our sleep hormone disrupting our sleep cycles. Taking tech breaks can bring more joy and purpose to your life by causing you to focus on the present moment. Short tech breaks allow you be more productive long-term. Try getting sunlight for a natural energy booster. Natural light helps to regulateour internal alarm clockcausing us to be more alert and energized during the day. Improving the quality of our sleep can also give us the energy we need to incorporate more movement into our daily lives. Although it may seem counterintuitive, exercising regularly doesn't wear us out- it actually increases our energy and makes us more productive. Ever heard the saying "motion creates positive emotion?" Exercise stimulates more blood flow and oxygen to the heart and brain helping to improve mood and confidence- all of which can directly increase performance and productivity. Exercise is also the number one way to reduce stress-another natural energy drainer. Reducing stress from the mind and body helps to make room for better thinking and a healthier body.

Resting, eating, and moving, are the best ways for enhancing performance and increasing productivity in all aspects of life. (Insert graphic below)

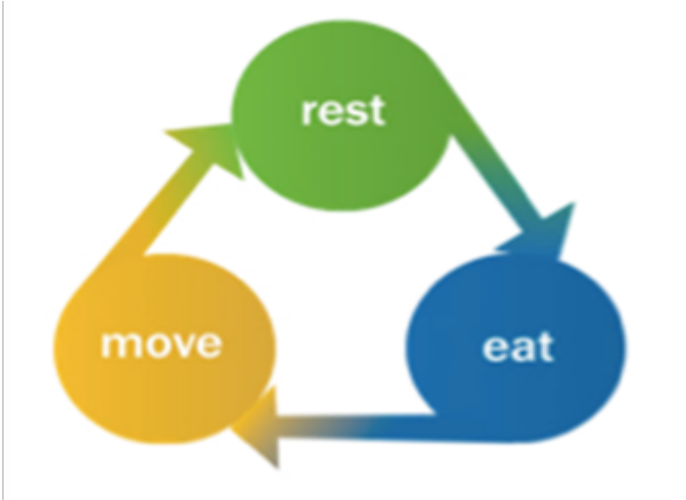


These three simple ingredients are what make up the On Target Living Lifestyle. This lifestyle program is centered on teaching people the benefits of rest and recovery, quality nutrition, and daily movement which ultimately leads to increased performance, decreased sick days, lost work time, lowered health care costs, and revitalized company culture.

Contact us atinfo@ontargetliving.com to learn more about how our team at On Target Living can educate, train, and empower you or your team to REST EAT MOVE.

\section{Acknowledgements}

None.

\section{Conflict of interest}

Author declares that there is no conflict of interest. 